\title{
Moderate coffee consumption associated with lower risk of mortality in prior Acute Coronary Syndrome patients: prospective analysis in ERICO cohort
}

\author{
Andreia Miranda $^{1}$, Alessandra Goulart ${ }^{2}$, Isabela Benseñor ${ }^{2,3}$, Paulo Lotufo $^{2,3}$ and \\ Dirce Marchioni ${ }^{1}$ \\ ${ }^{1}$ School of Public Health, University of Sao Paulo, Sao Paulo, Brazil, \\ ${ }^{2}$ Center for Clinical and Epidemiological Research, University Hospital, University of Sao Paulo, Sao Paulo, Brazil \\ and \\ ${ }^{3}$ School of Medicine, University of Sao Paulo, Sao Paulo, Brazil
}

\begin{abstract}
Coffee is one of the most widely consumed beverages around the world. Several studies have observed an inverse association between coffee consumption and all-cause mortality in population-based studies. Nevertheless, little is known about these associations in patient populations with a prior Acute Coronary Syndrome (ACS). To examine the association between coffee consumption and the risk of mortality in patients with a prior acute myocardial infarction (MI) or unstable angina. Data were from the longitudinal prospective study "Strategy of Registry of Acute Coronary Syndrome Cohort - ERICO". The cohort involved 1,085 patients diagnosed with ACS, between 2009 to 2013 . For this analysis we used data after 180 days until 4 years' follow-up, totalizing 928 participants. The coffee consumption was obtained using a questionnaire multiplying the reported frequency by the reported portion size. Subsequently was calculated the average of coffee intake (mL/day) and categorized (cups/day) into $\leq 1,1-3$, and $>3$. Information on mortality was ascertained by medical registries and death certificates. Cox regression models to estimate hazard ratios (HRs) for mortality according to coffee consumption adjusted for potential confounders were performed. Kaplan-Meier survival curves with the log-rank test were analyzed. Most patients $(99.0 \%)$ drank coffee, and the median total coffee intake was $125 \mathrm{~mL} / \mathrm{day}$. During a median follow-up of 4 years, a total of 111 deaths occurred, including 59 CVD-related and 24 MI-related deaths. Moderate coffee consumption was inversely associated with all-cause mortality. Participants who consumed 1-3 cups of coffee/day, showed an $81 \%$ lower risk of all-cause mortality than those who consumed $\leq 1 \mathrm{cup} /$ day (adjusted HR $0.19 ; 95 \% \mathrm{CI}$ : $0.11,0.34$ ). For patients with higher coffee consumption ( $>3$ cups/day), there was a positive association with mortality, with HR of 2.10 (95\%CI: 1.05, 4.22). Corresponding HRs were 0.22 (95\%CI: $0.11,0.48)$ for $1-3$ cups/day and 2.66 (95\% CI: $1.04,6.83)$ for $>3$ cups/day for CVD mortality; and 0.23 (95\%CI: $0.07,0.71)$ and $1.59(95 \% \mathrm{CI}: 0.25,10.0)$ for MI mortality, respectively. After stratification by smoking status the analysis revealed lower risk of all-cause mortality in never and former smokers drinking $1-3$ cups/day (HRs $0.10 ; 95 \%$ CI: $0.04,0.24$ and $0.18 ; 0.08$, 0.42, respectively). Among current smokers there was a positive association between $>3$ cups/day and mortality (HR $8.50 ; 95 \%$ CI: $1.18,16.35)$. The moderate consumption of coffee, impacted in a lower risk of all-cause, CVD and MI mortality in patients with a prior Acute Coronary Syndrome, particularly in nonsmokers.
\end{abstract}

\section{Conflict of Interest}

There is no conflict of interest. 